\title{
Investigación y formación de recursos humanos en ciencias sociales sobre la Amazonia*
}

Luis E. Aragón - Professor do Núcleo de Altos Estudos Amazônicos da Universidade Federal do Pará (NAEA-UFPA)

\section{Resumo}

Este texto trata do estado da arte da pesquisa em ciências sociais na Amazônia continental. O documento conclui que é difícil traçar um perfil da situação da pesquisa em ciências sociais aplicadas à Amazônia, na base dos dados disponíveis para o estudo. Com tudo, o estudo conseguiu identificar pelo menos 150 instituições que realizam pesquisa e produzem ciência e tecnologia relacionadas com a Amazônia nos oito países, das quais aproximadamente 100 fazem pesquisa de tipo social. Essas instituições estão concentradas principalmente no Brasil e na Colômbia.

\section{Abstract}

This is a study of the state of the art of the scientific research in social sciences in continental Amazonia. The study concludes that it is extremely difficult to define the situation of scientific research in social sciences with the data available for the study. Nevertheless the study was able to identify some 150 institutions that conduct research and produce science and technology related to the A mazon in the eight countries, of which approximately 100 conduct research on social sciences. Those institutions are located mainly in Brazil and Colombia.

\section{Palavras-chave}

Amazônia, Países amazônicos, Ciências Sociais e Humanas.

\section{Keywords}

Amazon; Amazonian countries; social and human sciences.

\footnotetext{
* Este trabajo se basa en documentos sobre el estado del arte de la investigación de ciencias sociales en la Amazonia brasileña, colombiana y peruana, preparados por consultores del Centro Internacional de Investigaciones para el Desarrollo (CIID) de Canadá (Aragón, 1990; Correa, 1990; Mora \& Zarzar, 1990). Materiales esparsos fueron utilizados para completar el anál isis en los demás países amazónicos. El documento aquí presentado forma parte del trabajo desarrollado por el autor como becario del Consejo Nacional de Desarrollo Científico y Tecnológico de Brasil. Agradecimientos a FLACSO/Ecuador por el apoyo logístico y bibliográfico recibido durante la elaboración de este documento. Nota del autor: Este trabajo fue elaborado durante 1992. Algunas cosas descritas en el texto han cambiado desde entonces, pero la mayoría de las informaciones permanece vigente.
} 
La investigación amazónica tiene raíces desde la época colonial, cuando países europeos, especialmente Inglaterra, Francia, Alemania, España y Portugal, mantenían naturalistas, cronistas y viajeros describiendo lo exótico de la región y coleccionando materiales de la fauna, la flora y la etnografía regionales. Los resultados de esas expediciones abarcan hoy un número representativo de volúmenes, escritos y colecciones de material biológico y etnográfico de significativo valor histórico (OLIVEIRA, 1983).

A partir de 1960 la investigación amazónica se intensifica, especialmente en Brasil. La Amazonia pasa a ser objeto específico de estudios profundos y detallados dentro de la propia región y fuera de ella. Durante la década de los setenta se desarrolla el proyecto radagramétrico en Brasil y Colombia, creando una importante base de datos para el conocimiento de los recursos naturales de la región. Durante estas últimas décadas también, numerosas tesis de maestría y doctorado, especialmente en universidades norteamericanas, fueron realizadas sobre la Amazonia. Por ejemplo, un levantamiento efectuado en 1977 de las tesis de Ph.D. reportadas a University Microfilms International por universidades de Estados Unidos y Canadá totalizó 6.000 tesis sobre América Latina y el Caribe producidas entre 1962 y 1977, para un gran total de 7.200 desde el início de la recolección en 1931. Algunas de esas tesis versan sobre la realidad amazónica, concentrándose principalmente en temas de antropología, geografía y agricultura (DEAL, 1977).

¿Cómo se presenta lo social en ese auge de investigación y cuál es su contribución para la producción científica y tecnológica y para el propio desarrollo de la región? Este trabajo busca identificar y analizar el estado de la investigación y la formación de recursos humanos en ciencias sociales relativos a la Amazonia, y lanzar algunas ideas sobre formas de cooperación que permitan desarrollar la ciencia social aplicable a la realidad amazónica.

Estas cuestiones conducen, primeramente, a enmarcar lo que se entiende por investigación social y como ella ha sido aplicada a la realidad amazónica. En ese sentido las recomendaciones de la Comissâo Coordenadora Regional de Pesquisa para a Amazônia, de Brasil (CORPAM, 1990) abarcan el universo de la investigación social en la Amazonia, según las cuales el proceso de ocupación de la región comprende un conjunto de condiciónes que delinean el perfil del desarrollo regional con problemas complejos de supervivencia humana, implantación de estructuras y procesos de trabajo que asumen características peculiares en la formación socioeconómica de la región, 
en que sistemas extractivos se transforman como resultado de la interacción compleja entre culturas, organizaciones sociales y procesos económicos. De esa manera se incorporan en la investigación socioeconómica los procesos de ocupación rural y urbana, los procesos productivos, las organizaciones sociales, la interacción hombre-medio ambiente, juntamente con los procesos de urbanización, de reforma agraria, habitación, procesos de trabajo, y las múltiples dimensiones de la organización social en general. Se incluye también en la investigación social la problemática de las poblaciones humanas, es decir, el examen de la heterogeneidad cultural, étnica y demográfica de la población de la región, las clases sociales que la componen y las relaciones de dominación y subordinación a que son sometidos sus habitantes, lo que implica análisis macro y micro de las poblaciones indígenas, caboclas, migrantes y demás grupos humanos que habitan la región, y sus articulaciones con las sociedades nacionales.

En esas bases, un balance sintético por país permitirá conocer el estado de la investigación y de la formación de recursos humanos en ciencias sociales aplicadas a la Amazonia. Naturalmente este balance está sujeto a la disponibilidad de informaciones extremamente escasas en la mayoría de países y la forma en que ellas son reproducidas. El orden de exposición obedece a la importancia que tiene en cada país la investigación amazónica en general, de acuerdo con las informaciones disponibles.

\section{BRASIL}

Hasta 1950, la producción de ciencia y tecnología en la Amazonia obedecía a los ciclos económicos y muy poco a la acción directa del Estado. A partir de este año se agudiza la ideología desarrollista del país y se institucionaliza una política científica y tecnológica, la cual se manifiesta, principalmente, con la creación del Conselho Nacional de Desenvolvimento Científico e Tecnológico (CNPq) y de la Coordenação de Aperfeiçoamento de Pessoal de Nível Superior (CAPES) en 1951 (BARROS \& BENEDITO, 1990, p. 28).

Aunque la repercusión de la política de desarrollo científico del país se haya sentido tardíamente en la Amazonia, la importancia estratégica y económica de la región determinó acciones específicas que contribuyeron para el avance de la investigación amazónica. Como reacción a la propuesta de la Organización de las Naciones Unidas para la Educación, la Ciencia y la Cultura (UNESCO) de crear el instituto internacional de la Hilea Amazónica en 1946, surgió, como propuesta 
del CNPq, el instituto Nacional de Pesquisas da Amazônia (INPA), en 1952, en Manaus, proyectándose hasta hoy como el instituto de investigación más importante de la Amazonia brasileña, especialmente en ecología y biología. Fue también a partir de 1950 que se multiplicaron las instituciones de ciencia y tecnología en la región. Las iniciativas aisladas como la del Museu Paraense Emílo Goeldi (MPEG) (1866), del Instituto Evandro Chagas (1936) y de la Faculdade de Ciências Agrárias do Pará (1945) y otras escuelas aisladas de odontología, medicina, química e ingeniería en Belém, y a la efímera existencia de la Universidade Livre de Manaus de 1906, se siguieron el establecimiento de universidades federales, comenzando por la de Pará en 1957, que integró la mayoría de escuelas aisladas funcionando en Belem, la de Amazonas en 1962, la de Maranhão en 1966, la de Acre en 1971 y, más recientemente, la de Rondônia en 1982, la de Roraima en 1990 y la de Amapá en 1991, y algunas universidades estatales.

Durante la década de los setenta la política de desarrollo de la Amazonia se torna más evidente, la cual va acompañada de una política expresa de ciencia y tecnología a través del Programa do Trópico Úmido (PTU) creado por decreto presidencial en 1972, para coordinar la contribución de la ciencia y la tecnología para el mejor conocimiento de las condiciónes de adaptación del ser humano a las peculiaridades del Trópico Húmedo Y a la preservación del equilibrio ecológico de la región amazónica (BARROS \& BENEDITO, 1990, p. 33).

El programa PTU tuvo gran repercusión en el avance de la investigación en la región, principalmente en el área de agricultura, debido a la filosofía de colonización del Programa de Integração Nacional (1970-1974). Más de $85 \%$ de los 431 proyectos financiados entre 1976 y 1986, período auge del programa, se iniciaron a partir de 1980 y aquéllos de carácter social, que representaban solamente $20 \%$ del total, comienzan prácticamente en su totalidad a partir de ese año (BENEDITO, 1990).

El programa PTU se suspende en 1986, resurgiendo en 1989, en la condición de secretaría ejecutiva de la CORPAM, órgano de asesoría vinculado al Ministério de Ciência e Tecnología. La CORPAM fue creada en ese año por ley del Congreso Nacional con el objetivo de definir una política de desarrollo científico y tecnológico apropiado para la Amazonia. A la CORPAM la integran representantes de organismos de investigación, universidades, investigadores, instituciones ambientalistas, instituciones de fomento y otros representantes, dando destaque especial a entidades e investigadores con sede en la Amazonia. 
Aproximadamente 84 unidades de investigación con sede en la Amazonia y 16 con sede en otras regiones brasileñas producen ciencia y tecnología sobre la región amazónica brasileña, pero solamente 19\% de aquellas localizadas en la Amazonia realizan investigación de tipo social, pese a que $33 \%$ de la demanda de información científica y tecnológica sea por ciencias sociales (AZEVEDO, 1989, p. 16-7).

El desarrollo de la investigación social y el número y calificación de los investigadores en esta área están todavía atrás en relación a las otras ramas del saber. Datos de la CORPAM de 1989 demuestran que solamente $20 \%$ (362) de los investigadores en instituciones amazónicas realizan investigación de tipo social y muestran el bajo porcentaje de doctores $(11,9 \%, 43$ del total) y de magisters $(30,6 \%, 111$ del total) actuando en investigación social en la Amazonia en relación al porcentaje de investigadores con esa titilación en la región, en otras ramas del saber (21,9\% y 52,5\%, respectivamente) (ARAGÓN, 1990).

Por el volumen de investigaciones y cantidad de investigadores en el área social, se destacan la Universidade Federal do Para (UFPA), el Museu Paraense Emílio Goeldi, la Universidade Federal de Mato Grosso (UFMT), el Instituto de Desenvolvimento Económico e Social do Pará (IDESP), la Universidade Federal do Acre y la Universidade do Amazonas. Los investigadores mejor calificados en ciencias sociales se encuentran en las universidades, especialmente en aquéllas que mantienen programas de postgrado, lo que no es sorprendente, pues los grupos de investigación están organizados alrededor de cursos de maestría y doctorado.

El hecho de las universidades de la región estar realizando cada vez más actividades de docencia, de investigación y de extensión relacionadas con asuntos amazónicos permite concluir que ellas están asumiendo su verdadera vocación amazónica. Es decir, asumiendo su identidad, no solamente por su ubicación geográfica, mas fundamentalmente por su relación con la problemática regional. La vocación amazónica es la contextualización de la universidad, es ejercer la universalidad del conocimiento científico a partir de la realidad concreta regional donde la universidad se inserte (VALENCIA GRANADA, 1988, p. 134). La universidad, así entendida, pasa de su condición de universidad en la Amazonia, y se convierte en verdadera universidad amazónica, pasando a convivir y ser parte integrante de la problemática y de las soluciones que la región enfrenta, hecho que le garante un espacio propio y le exige acciones concretas sobre los destinos de la región en aquello que más le compete: la generación y transmisión de ciencia, tecnología y cultura. 
Dos instituciones se destacan en la investigación social en la Amazonia brasileña: el Museu Paraense Emílio Goeldi y el Nucleo de Altos Estudos Amazônicos de la Universidade Federal do Pará (NAEA).

El Museu Paraense Emílio Goeldi fue creado en Belém el 6 de octubre de 1866, pero es a partir de 1894 que él asume status de institución científica, sobre la dirección del zoólogo suizo Emílio Augusto Goeldi, quien "con abundantes recursos oriundos de la producción de caucho" lo reestructuró y adaptó a las exigencias científicas de la época (FIGUEREDO ; BRUNI, 1987, p. 6). Su objetivo fundamental es

investigar la flora, la fauna, el hombre de la Amazonia en su ambiente físico, preservar y ampliar los respectivos acervos y dar subsidios, a través de los resultados de investigaciones y colecciones, a la realización de actividades de extensión y difusión científica y cultural (MPEG, 1989).

El Departamento de Ciencias Humanas del MPEG cuenta con 37 investigadores. Hasta 1980, los investigadores de este departamento (en su mayoría antropólogos), realizaron aproximadamente 120 trabajos de campo, con duración media de dos meses y publicaron cerca de 130 trabajos en la forma de monografías, conferencias y artículos abordando principalmente aspectos socioculturales, ergológicos y de contacto de grupos indígenas localizados en diferentes áreas de la Amazonia; sociedades rurales; aspectos regionales y administrativos; organización social y económica; sistemas de patronato y endeude; fiestas religiosas; y especulación inmobiliaria. A partir de 1980 las líneas de investigación del Departamento de Ciencias Humanas se amplían y diversifican, crece el numero de investigadores con titulación postgraduada y se amplían las relaciones ciencia-comunidad; los acervos etnográficos y arqueológicos son tratados técnicamente, llegando hoy a 14 mil piezas etnográficas y 2.734 elementos arqueológicos, y el acervo bibliográfico general fue ampliado totalizando hoy cerca de 171 mil títulos.

En febrero de 1973 el NAEA inaugura oficialmente sus actividades con el Curso Intemacional de Formação de Especialistas em Desenvolvimento de Áreas Amazônicas (FIPAM). La propuesta básica del NAEA era hacer del Núcleo un foco central, cristalizador, pero operativamente dinámico, de la misión universitaria. Sin perjudicar las funciones propias de los centros y departamentos, el NAEA sería la convergencia y la irradiación de la universidad en su afán de crear el saber tomando como materia prima la realidad amazónica, debiendo asumir, por lo tanto, la responsabilidad de generar conocimiento derivado de un trabajo interdisciplinar (MENDES, 1983, p. 2-3). 
El área de estudio del NAEA es la Amazonia continental (Panamazonia) y sus objetivos fundamentales son: la docencia a nivel de postgrado; la investigación científica, esencialmente la de naturaleza socioeconómica relacionada con la problemática amazónica; y la información científica, a través de la colecta, elaboración, procesamiento y divulgación de datos y documentos sobre la Amazonia.

Fue a través de grupos de estudio e investigación interdisciplinarios de los cursos FIPAM que el NAEA inició en la Amazonia y posiblemente en Brasil los estudios científicos sobre la expansión de la frontera amazónica, sobre la política y los modelos de desarrollo de la región, sobre urbanización y migración interna y sobre actividades de la economía campesina en la Amazonia.

Laboran en el NAEA más de 20 investigadores y/o profesores, principalmente antropólogos, sociólogos, economistas, geógrafos e historiadores, la mayoría con doctorado. Las investigaciones tratan de educación, cultura y religión, problemas agrarios, problemas urbanos, historia, medio ambiente, política científica y tecnológica, políticas públicas, trabajo y desarrollo, y migraciones.

Un punto importante a considerar en este estudio se refiere al tipo de investigación realizada, es decir, cuales son los temas mas frecuentes en los proyectos de investigación en curso. Los proyectos registrados por el Sistema de Informação Científica e Tecnológica da Amazonia (INFORMAM, 1987; 1988) reflejan la preocupación por mejorar la calidad de la docencia en la región, principalmente a través de las investigaciones sobre pedagogía y producción de material didáctico en la Universidade Federal de Mato Grosso y de los de psicología de la educación, comportamiento y psicología experimental en la Universidade Federal do Pará. No es sorprendente que los asuntos agrarios y urbanos, de penetración del capitalismo, fuerza de trabajo y asuntos indígenas ocupen un lugar de destaque en las propuestas de investigación social amazónica. Los procesos de ocupación del territorio amazónico, estimulados por políticas poco consistentes de desarrollo de la región, generaron conflictos fundiarios serios, muchos de los cuales violentos, expandiendo la concentración de la propiedad de la tierra y la especulación inmobiliaria, lo que estimuló, sin duda, muchos investigadores a dedicarse a investigar esa problemática. El impacto del NAEA es evidente en la realización de investigación en estos tópicos y la del MPEG en la investigación de los problemas indígenas.

Investigaciones en lingüística, letras y artes reflejan por un lado el trabajo antropológico, principalmente del MPEG, referente a investigación del lenguaje y dialectos de grupos indígenas y, por otro 
lado, la acción de varias universidades con programas de investigación sobre aspectos regionales del lenguaje. El sector de información científica y tecnológica refleja la propia acción del INFORMAM y de diversas propuestas de investigación en el área de bibliotecología, en la generación de estadísticas regionales y en algunos proyectos de formulación de política científica y tecnológica para la Amazonia.

Un tema prácticamente ausente en los registros del INFORMAM es el de estudios demográficos. Excepto por los trabajos de migraciones internas iniciados en el NAEA en 1976, el grueso de estas investigaciones se han concentrado fuera de la región amazónica, principalmente en la Universidade Federal de Minas Gerais y en la Universidade Federal de Rio de J aneiro (BERQUÓ, 1983; 1988). En el caso de migraciones, por ejemplo, el inventario de la producción técnico-científica sobre el tema en la Amazonia Legal, realizado bajo la coordinación de Donald Sawyer (1989), incluye cerca de dos mil referencias bibliográficas de documentos sobre el asunto, producidos en Brasil y en el exterior, y abarca el trabajo de aproximadamente 900 autores y coautores. Los trabajos fueron publicados en 23 países, siendo que Brasil abarca la mayoría (71 \%), $22,5 \%$ de estos fueron publicados en Rio de J aneiro, 12,7\% en São Paulo $11,3 \%$ en Pará. En otros tópicos de la demografía la investigación en la región es prácticamente nula.

Es importante mencionar que hay fuera de la región amazónica instituciones importantes que realizan investigación de tipo social sobre la Amazonia, entre las cuales se destacan además de la Universidade Federal de Minas Gerais, la Universidade Federal do Rio de J aneiro, el Museu Nacional do Rio de J aneiro, la Universidade de São Paulo, la Universidade de Brasília y el Instituto Sociedade, População e Natureza, de Brasilia.

Pese al relativo adelanto de la investigación amazónica en Brasil, la escasez de recursos humanos calificados en la región es tal vez la restricción mayor al desarrollo técnico-científico en la Amazonia. La región Norte de Brasil mantiene solamente 3,05\% (1.617) de los investigadores que laboran en el país (en 1986), siendo que, de ellos, escasamente $10 \%$ son doctores, representando menos de $1,5 \%$ de los investigadores con doctorado en el país (ARAGÓN, 1990).

Se pudiera decir que la política de desarrollo científico y tecnológico a través de la creación y fortalecimiento de programas de postgrado lato (especialización) y estricto senso (maestría y doctorado) fortaleció aún más los centros desarrollados del Centro-Sur, siendo que solamente a finales de la década de los setenta comienza a beneficiarse de manera significativa la región Norte. Como afirma Durham: 
es preciso reconocer que el desarrollo del postgrado, en Brasil, no derivó de un proceso espontáneo de crecimiento de la producción científica y del perfeccionamiento de la formación de sus cuadros, sino de una política deliberada del Estado. Ella obedecía a una decisión explícita, por parte del gobierno militar recién establecido [...]. Su objetivo es la modernización de la enseñanza superior en los cuadros del proyecto de desarrollo económico entonces adoptado (DURHAM, 1991, p. 101).

Actualmente las universidades y otras instituciones de educación superior de la región cubren prácticamente todas las áreas del saber a nivel de graduación. Existen (1991) en las 47 instituciones de educación superior localizadas en los estados de la Amazonia Legal (de las cuales 10 son universidades), 351 cursos de graduación, de los cuales $62,68 \%$ son en las áreas de ciencias sociales, ciencias humanas, lingüística, artes y letras. Los 351 cursos de la Amazonia representan 7,16\% de los 4.901 cursos de graduación existentes en el país y los de ciencias sociales, ciencias humanas, lingüística, artes y letras representan $7 \%$ del total de cursos en esas áreas en el país. Mas de $64 \%$ de los estudiantes matriculados en las instituciones de educación superior amazónicas cursan carreras en ciencias sociales, ciencias humanas, lingüística, artes y letras; y son estos cursos también los que muestran mayores porcentajes de conclusión de carreras $(74,91 \%)$, lo que indica gran potencial para la implantación de programas de investigación y estudio en esas áreas. Esas cifras siguen la tendencia nacional (BRASIL, 1992).

Iniciados en 1973, en el área de geociencias en la UFPA y de botánica en el INPA, existían en 1989, 28 cursos de postgrado stricto senso en la Amazonia, 22 de maestría y 6 de doctorado. Ese número de cursos de postgrado representaba escasamente $1 \%$ de los cursos de ese tipo existentes en Brasil (UFPA, 1989, p. 5). Siete de esos cursos, todos de maestría, eran en ciencias sociales y humanas, en las áreas de derecho, educación, planificación del desarrollo, letras y psicología.

Es importante notar que históricamente los cursos de postgrado en Amazonia estuvieron relacionados con las áreas de ciencias exactas y naturales. Hasta 1984, cuando se crea en la UFPA la maestría en derecho, el único curso de maestría en ciencias sociales era el de planificación del desarrollo, en el NAEA. Durante los últimos cuatro años, sin embargo, los cursos de maestría en ciencias sociales y humanas se amplían en la región, especialmente en el área de educación, al mismo tiempo que Belem y Manaus pierden la exclusividad de la educación 
postgraduada, con el surgimiento de los cursos de maestría de las universidades do Maranhão y Mato Grosso.

Los cursos más numerosos son los de derecho y de planificación del desarrollo. Hasta septiembre de 1989, habían defendido tesis 306 individuos, 280 a nivel de maestría y 26 a nivel de doctorado, siendo 39 en la maestría de planificación del desarrollo y 6 en la maestría de derecho.

Reconociendo que el proceso de formación de investigadores solamente culmina con el doctorado, considerando la necesidad de profesionales altamente calificados en la Amazonia y tomando en cuenta la experiencia del NAEA en la realización de investigación y educación postgraduada de carácter interdisciplinar, interinstitucional e internacional, el NAEA establecerá un programa de doctorado en el futuro próximo².

El doctorado del Núcleo deberá identificar y profundizar la problemática relativa al desarrollo armónico y sostenido de las regiones del trópico húmedo, llevando en cuenta sus carácterísticas históricas y ambientales, y generar conocimientos teóricos y empíricos nuevos capaces de abarcar la realidad particular de esas regiones y de entender los mecanismos de su desarrollo. Es decir, contribuir para la generación de conocimiento nuevo sobre la realidad del trópico húmedo, en general, y de la Amazonia, en particular, basado en la problemática del desarrollo sustentado o ecodesarrollo. En otras palabras, buscar medios para satisfacer las necesidades materiales básicas de la población regional a través de la gerencia responsable de sus recursos naturales y sin comprometer la capacidad de generaciones futuras de vivir en armonía con el medio ambiente. En fin, deberá estimular a la reflexión y análisis profundos sobre, para qué y para quién se orientan la producción de nuevo conocimiento y modelos alternativos de desarrollo del trópico húmedo.

\section{PERU}

La investigación amazónica en general en Perú sigue la visión estatal de la Amazonia como despensa del país. Esta visión ha hecho que la Amazonia peruana haga parte de diversos programas de integración, colonización y explotación de recursos naturales a lo largo de la historia contemporánea del país y que el rol que, en esas políticas

\footnotetext{
${ }^{2}$ A partir de una recomendación durante la III Asamblea General de la UNAMAZ, realizada en Manaus en junio de 1992, el NAEA establece el Curso de Doctorado en Desarrollo Sostenible en los Trópicos Húmedos, a partir del segundo semestre de 1994.
} 
buscó ocupar la universidad, parece haber sido el de dotar a los proyectos ya diseñados de conocimientos técnicos y personal capacitado necesarios para su implementación (MORA, 1988, p. 321).

Que las cuatro universidades localizadas en la Amazonia peruana (fundadas después de 1960) surgieron como instituciones vinculadas a programas de colonización impuestos por el poder central, lo demuestran las carreras estrictamente técnicas con que ellas iniciaron sus labores: agronomía, ingeniería forestal, ingeniería de alimentos. Los aspectos sociales fueron poco considerados.

Hay cerca de 27 instituciones en el país que adelantan investigaciones y/o tienen producción científica en ciencias sociales relacionadas con la Amazonia, la mayoría localizadas en Lima. Aquellas localizadas en la región amazónica aún hoy tienen poquísimos programas en el área de ciencias sociales (MORA ; ZARZAR, 1990).

La investigación social sobre la Amazonia peruana surgió tardíamente, especialmente a partir de los últimos 25 años, más por iniciativa personal de algunos investigadores que por programas institucionales o estatales. Eso explica por que todavía la gran concentración de investigaciones en curso (17 de 80) sea realizada por investigadores autónomos, o no asociados a ninguna institución. Las investigaciones y publicaciones se concentran en antropología, sociología, historia, e investigación en lingüística (MORA; ZARZA, 1990, p. 13).

La institucionalización de la antropología en el Perú se da durante la década de los cuarenta, con la creación del Instituto de Etnología y Arqueología de la Universidad Mayor de San Marcos (Lima) en 1946, pero las primeras referencias importantes de antropología amazónica surgen solamente durante los últimos 25 años, centradas en el estudio y conocimiento de las culturas indígenas de la región.

Los investigadores autónomos y los del Centro de Investigación y Promoción Amazónica (CIPA) han contribuido de manera decisiva en el desarrollo de la sociología amazónica, especialmente en lo que concierne a políticas de desarrollo regional, ampliación de la frontera, colonización y problemas de las poblaciones humanas enfrentados por la región (MORA ; ZARZA, 1990).

El levantamiento realizado por Santos Granero (1991) compiló 161 referencias históricas relativas a la Amazonia, publicadas en el Perú y en el exterior. De estas, 2,5\% fueron publicadas en la década de los cincuenta, $6,8 \%$ en la década de los sesenta, $28,1 \%$ en la década de los setenta y $61,2 \%$ en la década de los ochenta. Según ese autor: 
Junto con el aumento del número de trabajos realizados, se percibe una mayor sofisticación de los mismos, como consecuencia de una revisión más exhaustiva de las fuentes clásicas, de la búsqueda de nuevos documentos, del uso de instrumentos metodológicos más refinados y de un mayor rigor académico (SANTOS GRANERO, 1991, p. 51).

Pese al carácter histórico o historiográfico de las referencias revisadas por Santos Granero (1991), solamente 10\% de los 95 autores peruanos registrados en el levantamiento son historiadores profesionales; en total, $60 \%$ de los textos son responsabilidad de antropólogos. El avance de la antropología lleva a ocupar espacios libres dejados por ramas del saber no todavía bien desarrolladas o interesadas en cuestiónes amazónicas.

Lo importante de ver es el avance que se consigue en el tratamiento histórico de la cuestión amazónica a partir de finales de la década de los sesenta, cuando se deja de lado la descripción epopeyica de la historia oficial para centrarse en la realidad política y social de los grupos humanos que habitan la región, principalmente indígenas. Esos trabajos llevaron a análisis detallados de las relaciones comerciales, políticas y sociales entre sierra y selva; de las redes de intercambio existentes entre grupos indígenas de la propia Amazonia como entre éstos e indígenas de la sierra; así como al estudio de las formas de dominación predominantes en la Amazonia en la época de la colonia; las misiones; Ias doctrinas y las encomiendas. Otros tópicos importantes tratados por la producción científica en historia de la Amazonia peruana incluyen análisis de las formas de resistencia y rebeliones indígenas; de las políticas de incorporación de la Amazonia a la economía nacional desde la época de la colonia; y la identificación de los ciclos y frentes económicas de expansión experimentados por la región desde el período colonial. Finalmente Santos Granero (1991) identifica los más importantes vacíos en la historiografía amazónica, como por ejemplo la escasez o casi nulidad de estudios demográficos, la exagerada proporción de estudios que enfocan la problemática indígena - en comparación a otros grupos humanos de la región -, y el prácticamente exclusivo carácter nacional de los estudios, que difícilmente analizan la realidad amazónica peruana en un contexto panamazónico, internacional o mundial.

La investigación lingüística se limita, en la Amazonia peruana, casi que exclusivamente a los aspectos gramaticales de los idiomas vernáculos de la región. Este tipo de investigación es desarrollado principalmente 
por el Instituto Lingüístico de Verano, que ha investigado por lo menos 20 idiomas amazónicos, y por el Centro de Investigación de Lingüística Aplicada de la Universidad Mayor de San Marcos, que trabaja también sobre idiomas de grupos indígenas amazónicos pero en menor número (MORA; ZARZA, 1990).

Es importante mencionar otros tópicos también tocados por la ciencia social en el caso de la Amazonia peruana. Dada la importancia que los problemas ambientales han adquirido, surgieron algunas publicaciones o propuestas de investigación relacionadas con esos aspectos. Estudios económicos han enfatizado el problema de la coca y el narcotráfico, problemas de crédito agrario, economía extrativista y, junto con trabajos en sociología, estudios sobre ciclos económicos, colonización, economía de subsistencia y políticas de desarrollo regional.

Gracias a investigaciones realizadas en el CIPA, Ios estudios jurídicos vinculados a la problemática amazónica, especialmente indígena, están tomando fuerza durante la última década. Como resultado de este esfuerzo, existe ya una recopilación y sistematización de las normas legales producidas por el Estado peruano en el período 1821 1990, relacionadas con diferentes aspectos de la Amazonia (MORA; ZARZA, 1990).

Finalmente cabe destacar algunas iniciativas en el área de educación bilingüe, desarrolladas por el Proyecto de Educación Bilingüe del Alto Napo, el Instituto Superior Pedagógico de Loreto y la Universidad Nacional de la Amazonia Peruana. Los dos primeros buscan alterativas a la actividad desarrollada por el Instituto Lingüístico de Verano en la Amazonia, y en ese sentido se centran en conocer los sistemas de educación en las comunidades nativas, capacitación de maestros bilingües y maestros indígenas y en la elaboración de currículos alterativos para escuelas bilingües (MORA ; ZARZA, 1990). La experiencia de la Universidad Nacional de la Amazonia Peruana se centra en la formación de maestros bilingües en la propia universidad o en los locales de origen de las comunidades (BEDOYA, 1989).

Con referencia a la formación de recursos humanos en ciencias sociales aplicadas a los estudios amazónicos, es importante destacar que, a pesar de existir en el momento cuatro universidades nacionales y un instituto de investigaciones amazónicas en la Amazonia peruana (Universidad Nacional de la Amazonia Peruana, en Iquitos; Universidad Nacional Agraria de la Selva, en Tingo María; Universidad Nacional de San Martín, en Tarapoto; Universidad Nacional de Ucayali, en Pucallpa; e Instituto de Investigaciones de la Amazonia Peruana, en Iquitos), los programas de formación e investigación en ciencias sociales en esas 
instituciones son prácticamente inexistentes, excepto por algunas disciplinas como antropología, economía y sociología, que se puedan dar como parte del currículo de carreras como medicina y enfermería en Ia Universidad Nacional de la Amazonia Peruana, o recursos naturales de la Universidad Nacional Agraria de la Selva.

A nivel de pregrado el interés por los estudios amazónicos se da en facultades de ciencias sociales de las universidades del resto del país, especialmente en la de antropología de la Mayor de San Marcos y la Pontificia Universidad Católica del Peru. A nivel de postgrado la iniciativa corre por cuenta del propio estudiante del país o del exterior en hacer sus tesis sobre la Amazonia.

Esta falta de programas de formación de recursos humanos en ciencias sociales aplicables a la realidad amazónica contrasta con ese tipo de programas en otras ramas del saber, especialmente en ciencias agrarias, forestales, ingeniería de alimentos y ciencias biológicas. Como consecuencia, no es de esperar que sean los científicos sociales los que lideren o inclusive los que integren los grupos que definen las estrategias de desarrollo de la región. Sólo en años muy recientes se han llamado a científicos sociales a participar de dichos grupos. "Es un requisito más aparente que real Ilamarlos a conformar los equipos de planificación del desarrollo, porque, en realidad, nadie sabe bien para que se los llama ni qué importancia puede tener su opinión" (CHIRIF, 1991, p. 271). Esta falta de participación más activa de los científicos sociales deja ver claramente que aún se entiende en los medios oficiales que la cuestión del desarrollo amazónico se puede resolver de una manera simplemente técnica. Por otro lado, como agrega Chirif (1991), los científicos sociales en general no han conseguido responder de manera adecuada al desafío de la planificación del desarrollo, limitándose, salvo contadas excepciones, a realizar inventarios y diagnosticos, generalmente en base de datos secundarios que servían de base para una formulación técnica posterior. Obviamente, muchas de estas limitaciones se deben precisamente a la falta de programas de formación de recursos humanos especializados que puedan desempeñarse de manera mas eficiente en asuntos que se relacionan con el desarrollo de la Amazonia.

\section{COLOMBIA}

El interés por la investigación amazónica en general y por la investigación de tipo social en particular está asociada a instituciones relativamente antiguas en el país, como la Universidad Nacional, fundada en 1867, el Instituto Geográfico Agustín Codazzi (IGAC), fundado en 
1931, y el Instituto Colombiano de Antropología, fundado en 1941 (ALZATE ; RAMÍREZ, 1991); naturalmente, sin remontarse a las expediciones de Humboldt del siglo XIX o de las exploraciones europeas en épocas anteriores.

La presencia de la Universidad Nacional en la investigación amazónica se remonta a la década de 1920 con proyectos de ingeniería (Correa, 1990). El esfuerzo personal ejercido por profesores e investigadores de la Universidad Nacional en los diferentes departamentos, especialmente de antropología, agronomía, geografía y ciencias naturales ha sido constante a lo largo de los años. En la década de los setenta, se organiza el Programa Orinoquia-Amazonia (ORAM), que coordinaría un buen número de profesores, investigadores y estudiantes de la universidad interesados en investigación en la Amazonia y Orinoquia, el cual culmina en 1985 con la edición del último numero de su boletín. La investigación amazónica en la Universidad se fortalece durante la década de los ochenta y la presente, con la reactivación del Instituto Amazonico de Investigaciones (IMANI) de Leticia, después de 20 años de inercia, la estructuración de los proyectos de investigación en la Amazonia en un único modelo (Modelo de Desarrollo Amazonico, MODA), y con el establecimiento de dos mecanismos dinamizadores de la investigación amazónica: el Comité Amazónico en 1988 y el Instituto de Estudios Ambientales en 1990.

EI IGAC es responsable por la cartografía del país, el catastro y levantamientos geográficos, entre otras responsabilidades de investigación. Desde su propio nacimiento, el Agustín Codazzi ha acompañado la investigación amazónica, pero su mayor impacto se dio en la década de los setenta con el programa radagramétrico, que se realizó en convenio con el gobierno holandés con el objetivo de hacer un levantamiento y evaluación de los principales recursos naturales y condiciones socioeconómicas de la cuenca amazónica colombiana, y obtener la información básica para que el gobierno nacional pudiera planificar en forma integrada el desarrollo del sector suroriental del país, para incorporarlo al resto de la economía nacional (ALZATE ; RAMÍRES, 1991, p. 91).

Diversos proyectos especialmente referentes al uso de recursos naturales, cartografía, información geográfica y sensores remotos mantienen activa la presencia de ese Instituto en la Amazonia colombiana. En convenio con la Universidad Pedagógica y Tecnológica de Çolombia, de Tunja, el IGAC mantiene un curso de maestría en geografía .

${ }^{3}$ Este programa es actualmente ofrecido por el Departamento de Geografía de la Universidad Nacional de Colombia. 
El Instituto Colombiano de Antropología se funda bajo la tutela del célebre antropólogo francés Paul Rivet (Correa, 1990, p. 40). A lo largo de sus 50 años ha conseguido consagrarse a nivel intemacional por la seriedad de sus estudios, especialmente de su revista de antropología. Entre sus investigaciones se destacan trabajos de lingüística, demografía, medicina tradicional, etnología y etnografía de diferentes grupos indígenas colombianos.

Durante la década de los setenta se expande la investigación amazónica en Colombia. Se funda la Corporación Colombiana para la Amazonia en 1977, con el objetivo específico de promover la investigación en la Amazonia y Orinoquia, para el mejor conocimiento de sus recursos naturales, su flora, su fauna, así como la experimentación y difusión de tecnologías apropiadas al uso de esos recursos, y adelantar estudios, programas y proyectos para promover el desarrollo económico y social de esas regiones. Para desarrollar sus proyectos establece subsedes en Araracuara (Amazonas), Puerto Inírida (Guainía), San J osé del Guaviare (Guaviare) y Sierra de la Macarena (Meta) ${ }^{4}$. Durante esa década también se fortalecen los programas de investigación antropológica y lingüística de la Amazonia en la Universidad de los Andes, se crea la facultad de diplomacia en la Universidad J orge Tadeo Lozano y se inicia el programa Expedición Humana en la Universidad J averiana (CORREA, 1990).

A proximadamente 60 instituciones nacionales públicas o privadas y 15 extranjeras hacen investigación amazónica en Colombia (ALZATE \& RAMÍREZ, 1991). En relación a otras ramas del saber, las ciencias sociales están en ventaja en la investigación amazónica, tanto en términos del número de investigaciones, número y calificación de los investigadores $Y$ del número de instituciones que se dedican a este tipo de investigación.

Más de 56\% (119) de las 354 investigaciones amazónicas identificadas por Correa (1990) en el período 1984-1988 son de tipo social y 45 de las 53 instituciones que realizaban investigación amazónica en 1990, identificadas por ese autor, tenían proyectos de ciencias sociales, destacándose la Universidad Nacional, la Universidad de los Andes, la Corporación Colombiana para la Amazonia, el Instituto Lingüístico de Verano y el Instituto Colombiano de Antropología.

La infraestructura de investigación en la Amazonia colombiana es extremamente escasa, ya que se limita prácticamente a la Universidad de la Amazonia y al Instituto Colombiano Agropecuario, en Florencia, y

${ }^{4}$ La Corporación Colombiana para la Amazonia fue extinta dando origen al actual Instituto Amazónico de Investigaciones Científicas (SINCHI). 
al Instituto Amazónico de Investigaciones de la Universidad Nacional, en Leticia. Es importante destacar el trabajo desempeñado por investigadores autónomos en ciencias sociales, y el enfoque social de las investigaciones realizadas por las Secretarías de Salud de los departamentos de Guainía y Vaupés.

Fueron identificados, en total, 196 investigadores laborando en investigación social amazónica en Colombia, durante 1980-1990, que representarían aproximadamente $54 \%$ del total. De acuerdo a la calificación, la mayoría de investigadores que se dedican a la problemática amazónica en Colombia posee solamente la graduación, tanto en ciencias sociales como en las demás ramas del saber $(52,5 \%$ y $51,7 \%$ respectivamente), aunque a nivel de doctorado las ciencias sociales presentan una mayor concentración (14,7\% vs. 11,9\%) (CORREA, 1990, p. 149).

La mayoría de investigaciones cubre temas de antropología (38,5\%) y lingüística (20,5\%). Obviamente, se nota la acción del Instituto Colombiano de Antropología, del Departamento de Antropología de la Universidad de los Andes y del Instituto Lingüístico de Verano. Las únicas temáticas que se aproximan a esas dos son planificación regional y urbana $(11,8 \%)$ y economía $(7,5 \%)$, como resultado probablemente de agencias gubernamentales. Historia, sociología y artes totalizan un poco más de $12 \%$ y el resto cubre tópicos de arquitectura, arqueología, ciencias de la información, educación, ciencia política, comunicación, trabajo social, filosofía y geografía (CORREA, 1990, p. 154).

Se podría concluir diciendo que la investigación amazónica en Colombia, especialmente en ciencias sociales, está vinculada a universidades y otras instituciones que realizan diferentes tipos de investigación, donde por la fuerza de cierto número de investigadores se impone. De este esquema sale la Corporación Colombiana para la Amazonia, que desde su fundación fue creada específicamente para realizar investigación amazónica. El acervo bibliográfico acumulado por esa Corporación sobre la realidad amazónica puede ser en este momento el mayor del país. A nivel universitario, algunos departamentos como el de antropología en los Andes o los de geografía y antropología en la Nacional han de cierto modo liderado la investigación social en esas instituciones. Hay que destacar finalmente el esfuerzo que la Universidad de la Amazonia, de Florencia, viene realizando para sistematizar sus investigaciones en su propio Instituto Amazónico de Investigaciones (IAMI). A nivel nacional, el Instituto Colombiano de Investigaciones Científicas Francisco J osé de Caldas (COLCIENCIAS) viene haciendo esfuerzos desde 1986 para coordinar las acciones de todas las 
instituciones relacionadas con la investigación amazónica a través del Comité Nacional de Investigaciones sobre Amazonia (CONIA). Ese Comité busca orientar todas las investigaciones de la región a fin de coordinar acciones gubemamentales en materia de políticas de investigación para la región (VALENCIA GRANADA, 1988).

La formación de investigadores en asuntos amazónicos queda, en Colombia, por cuenta de algunos cursos de graduación de las universidades, que incluyen asignaturas que tocan la realidad amazónica y las posteriores monografías de conclusión de curso, por interés del estudiante. Los cursos de postgrado siguen el mismo destino, sin existir en el país una capacitación dirigida para la Amazonia ${ }^{5}$. La propia Universidad de la Amazonia solamente ahora está adecuando sus currículos para disciplinas más acordes con la realidad regional.

La mayoría de investigadores del país interesados en asuntos amazónicos ha sido formada en el exterior. Sin embargo, con la infraestructura existente en el país, la cantidad y calificación de investigadores en ciencias sociales especializados en Amazonia, Colombia tiene las condiciones para crear programas de pre y postgrado, aunando esfuerzos de varias instituciones.

\section{ECUADOR}

Como afirma Yánez (1991, p. 97), “hasta hace pocos años el estudio de la Amazonia ecuatoriana fue tarea casi exclusiva de los investigadores extranjeros". Entre los estudios realizados por extranjeros se destacan las investigaciones antropológicas de Rafael Kartzen sobre los J íbaros en 1935 y de Alfred Metraux sobre los indios del medio y alto Amazonas en 1948. Un paso importante para cambiar esta realidad se da con la creación del Departamento de Desarrollo Fronterizo y Asuntos Amazónicos en el Ministerio de Relaciones Exteriores del Ecuador, en 1982, que, instituido con la finalidad de sistematizar programas de cooperación para el desarrollo de la Amazonia ecuatoriana, realiza una serie de investigaciones y diagnósticos en diferentes áreas con financiamiento principal de la Organización de los Estados Americanos (OEA) a fin de subsidiar los programas de cooperación a ser desarrollados, entre los que se destacan algunos de cooperación bilateral con Colombia (ZUQUILANDA DUQUE, 1988).

${ }^{5}$ Actualmente se organizan cursos sobre asuntos amazónicos tanto en el Instituto Amazónico de Investigaciones (IMANI) de la Universidad Nacional, en Leticia, como en la Universidad de la Amazonia, en Florencia. 
Además del Departamento de Desarrollo Fronterizo y Asuntos Amazónicos del Ministerio de Relaciones Exteriores, hay una serie de entidades gubernamentales relacio-nadas con el desarrollo de la región amazónica ecuatoriana, las cuales realizan o encomien-dan investigaciones sobre planes y programas para el desarrollo en sus sectores de actuación. Entre estas se destacan el instituto Ecuatoriano de Reforma Agraria y Colonización, el Programa Nacional de Regionalización Agraria, el Fondo de Desarrollo Rural Marginal, el Programa de Desarrollo para el Sur del Ecuador, el Instituto Ecuatoriano de Recursos Hidráulicos, y el Centro de Reconversión Económica del Azuay, Cañary Morona Santiago, además de diferentes ministerios, entre los que se destaca el Ministerio de Energía y Minas, a cuyo cargo está la explotación petrolera en la Amazonia.

La producción científica amazónica en las universidades y escuelas politécnicas del país es incipiente, pese a la política del Consejo Nacional de Ciencia y Tecnología de:

avanzar gradualmente hacia una mayor autonomía en las decisiones tecnológicas y ampliar la capacidad nacional de investigación y desarrollo, estableciendo condiciones favorables para la transferencia, utilización, adaptación y creación del conocimiento que el país experimentará en el largo plazo y del respeto de la formación y creatividad de los investigadores, ingenieros y técnicos ecuatorianos (ZUQUILANDA DA DUQUE, 1988, p. 238).

Entre las universidades y escuelas politécnicas tanto públicas como privadas que se destacan en investigación, pero principalmente en actividades de extensión universitaria en la Amazonia, están la Universidad Nacional de Loja, la Universidad Particular de Loja, la Universidad Católica de Cuenca, la Escuela Politécnica Superior del Chimborazo, la Escuela Politécnica Nacional, la Pontificia Universidad Católica del Ecuador y la Universidad Central del Ecuador (ZUQUILANDA DUQUE, 1988).

La Universidad Nacional de Loja, en la ciudad de Loja, es una de las más antiguas del país. Actualmente posee ocho facultades y 32 carreras. Mantiene tres centros de educación en la Amazonia ecuatoriana: el Instituto Tecnológico de Yamzatza, con cursos de corta duración en ciencias agropecuarias; el Centro Universitario en Zumba (Chinchipe), con cursos de docencia para profesores de escuela primaria; y el Centro de Investigaciones en Padmi (Zamora). Se han realizado varias tesis de 
conclusión de curso sobre Amazonia, cubriendo temas como cultivo del cacao, parásitos del cultivo de la naranjilla, adaptación de especies forestales, adaptación de gramíneas y forrajeras, mejoramiento de suelos, control de plagas, identificación de maderas y otras. Está en estudio el establecimiento de un Centro de Estudios Amazónicos, que integre de manera interdisciplinar los estudios e investigaciones que la universidad realiza en sus diferentes unidades con relación a la Amazonia.

La Universidad Técnica Particular de Loja, con sede en Loja, realiza desde 1976 programas de extensión en Zamora, con carreras de contaduría pública y construcciones viales.

La Universidad Católica de Cuenca, con sede en Cuenca, mantiene desde 1974 una unidad de extensión en Méndes, provincia de Morona Santiago, donde ofrece carreras de medicina veterinaria y zootecnia, a un total actual de 150 estudiantes. Por la misma época fue establecido en la misma ciudad un colegio de ciclo diversificado. Posteriormente se ofrecen las carreras de educación e ingeniería empresarial en Macas, para un total actual de 300 estudiantes.

La Escuela Superior Politécnica del Chimborazo, con sede en la ciudad de Riobamba, fue creada en 1972 y posee en la actualidad dos unidades de extensión en Puyo: el Centro Experimental de Fátima y la extensión de la Facultad de Zootecnia en Río Negro. El primero realiza investigaciones sobre cultivos alternativos, domesticación de animales y capacitación de comunidades indígenas. El segundo realiza investigaciones sobre manejo de fauna silvestre y ganadería.

La Escuela Politécnica Nacional, con sede en Quito, realiza investigación sobre Amazonia a través del instituto de investigaciones Tecnológicas y las escuelas de hidráulica, biología y química. Los trabajos más importantes son en el área de petroquímica, clasificación y utilización de fármacos y desarrollo potencial hidroeléctrico de los ríos del Oriente ecuatoriano.

La Universidad Católica del Ecuador, con sede en Quito, desarrolla investigaciones en el área de biología, destacando la taxonomía vegetal y animal, la ecología de insectos polinizadores, la ecología de frugívoros y la ecología de los caimanes.

La Universidad Central del Ecuador, con sede en Quito, creó en 1980 la Comisión Multidisciplinar de Estudios Amazónicos, la cual trabajó intensamente hasta 1985. Durante ese período fueron producidos estudios sobre la Amazonia, destacando monografías sobre Morona, Napo, Nambija, Loja y Zamora. En 1991 se crea en esa universidad la Comisión de Estudios Ambientales, presidida por el rector e integrada 
por investigadores de varias facultades. La universidad viene desarrollando investigaciones sobre recuperación de zonas protegidas, bosques y cultivos amazónicos, navegabilidad del río Napo y tecnologías alternativas. Está en proyecto la creación de un Centro de Estudios Amazónicos de carácter interinstitucional, con la participación de algunas entidades gubernamentales, la Subsecretaría de Medio Ambiente del Ministerio de Minas y Energía, universidades y otros organismos.

Hay otras entidades que también realizan investigación amazónica, como la Fundación Natura, creada en 1978, en el área de biología y ecología, el Instituto Nacional de Investigaciones Agropecuarias del Ministerio de Agricultura y Ganadería, en el área de agricultura, ganadería y veterinaria, y el Instituto Geográfico Militar, en las áreas de cartografía y geografía en general. Este instituto mantiene el Curso Internacional de Especialización en Estudios e Investigaciones Geográficas, con fondos del Instituto Panamericano de Geografía e História, organismo de la OEA.

Es palpable la falta de investigación social en las entidades mencionadas. La investigación se centra en áreas técnicas o de utilización de recursos naturales, descuidando casi por completo el área social. A partir de 1981, cuando se crea el Centro de Documentación e Información de los Movimientos Sociales del Ecuador (CEDIME), organismo no gubernamental, la investigación en ciencias sociales en la Amazonia ecuatoriana se fortalece. A partir de 1988, el CEDIME crea una área específica para tomar cuenta de las cuestiones amazónicas en Ecuador. Esta área tiene como objetivos realizar actividades de investigación y acción en la región, a través de una línea de estudios, un trabajo de documentación, una participación sistemática en eventos de denuncia e información sobre problemas regionales, y la producción de materiales educativos para la sociedad regional y nacional, tendientes a crear conciencia sobre la realidad amazónica. Sus temas de investigación se centran en el análisis de las políticas estatales de ocupación de la región, en identificar y analizar las múltiples relaciones de los actores sociales que actúan en la región, los movimientos sociales, los ciclos económicos, el mercado, el medio ambiente, las poblaciones amazónicas, la problemática indígena y la situación de la mujer y los niños de la Amazonia. Además de investigación, el CEDIME viene realizando actividades con el objetivo de concretizar la delimitación y legalización de territorios indígenas, la identificación de alterativas productivas para colonos e indígenas amazónicos, además de estudiar alternativas para el uso del bosque y los impactos de la actividad petrolera en la región. Con el afán de analizar de manera crítica la situación de la Amazonia y 
difundir el conocimiento, el CEDIME ha establecido un programa de eventos científicos a nivel internacional, las J ornadas Interaccionales Amazónicas, con participación de investigadores de todos los países amazónicos. Las primeras fueron realizadas en julio de 1990 y las segundas en enero de 1992. Las dos actividades generaron un acervo bibliográfico importante sobre la Amazonia en general, destacando temas sociales. Además de las J ornadas, el CEDIME ha participado y organizado varios eventos nacionales en Quito y en ciudades amazónicas ecuatorianas.

El fortalecimiento de las ciencias sociales aplicadas a la problemática amazónica se concretiza en Ecuador con el establecimiento de las actividades de postgrado en estudios amazónicos de la Facultad Latinoamericana de Ciencias Sociales (FLACSO). Esa facultad es un organismo internacional de carácter regional y autónomo, creado en 1957, por países de América Latina y el Caribe.

Su creación surgió de la necesidad de generar una reflexión científica propia sobre América Latina y, a la vez, contribuir a la búsqueda de propuestas para el desarrollo de la región, mediante actividades de docencia de postgrado, de investigación y de cooperación técnica, en el ámbito de las ciencias sociales (FLACSO, 1991a, p. iii).

La sede ecuatoriana fue creada en 1975 por acuerdo con el gobierno ecuatoriano, mediante el cual se le garantiza a la facultad autonomía académica y administrativa. Siguiendo sus propósitos, FLACSO se ha empeñado en fijar las ciencias sociales en Ecuador a través de programas de docencia, investigación y cooperación técnica. Pocos años después de su establecimiento, ella da inicio a programas de postgrado: maestría en ciencias sociales con mención en estudios del desarrollo (1978-1980 y 1981- 1983), maestría en historia andina (19831985 y 1989-1991), maestría en ciencias políticas (1989-1991), maestría en economía (1990-1991), diploma superior en ciencias políticas con mención en asuntos latinoamericanos (1987-1988), diploma superior en ciencias sociales con mención en estudios amazónicos (1989-1990), maestría en ciencias sociales con mención en estudios amazónicos (19911992), maestría en antropología con mención en antropología andina (1991-1992), diploma superior en antropología con mención en antropología andina (1991-1993), curso de especialización sobre mujer, cambio social y desarrollo (1991), curso de especialización en diseño y gestión de proyectos de desarrollo forestal participativo en los Andes (1991) (FLACSO, 1991a). 
A partir de 1987, FLACSO/Ecuador se reorganiza dando énfasis a los programas de maestría. Dentro de esta nueva tendencia, en 1989 se incluye un programa de postgrado en estudios amazónicos con un año de duración, inicialmente para profesionales ecuatorianos (FLACSO, 1991 b). Con la experiencia del diploma de estudios superiores, se inicia la maestría en ciencias sociales con mención en estudios amazónicos, a partir de marzo de 1991, diseñada para dos años y ampliada para una clientela internacional. Cursan la maestría estudiantes provenientes de Ecuador, Colombia, Bolivia, Brasil, Perú y otros países de América Latina. Esta maestría esta diseñada para ampliar la comprensión que el estudiante tiene de los complejos problemas sociales de la Amazonia y capacitarlo, a través de un programa comprehensivo de materias de varias disciplinas y una especialización temática, mediante cursos sobre las condiciones particulares de esa región. El curso de maestría hace parte de las actividades de la unidad académica de estudios interdisciplinarios, e incluye asignaturas de economía, antropología, ecología, desarrollo regional, historia y sociología de la Amazonia, además de disciplinas de teoría y metodología de las ciencias sociales.

El perfil de los estudiantes de FLACSO es diversificado: en general, provienen de una formación básica en ciencias sociales, pertenecen principalmente a centros universitarios o de investigación, y de organismos públicos, o son profesionales liberales. La mayoría es ecuatoriana, pero hay estudiantes de otros países latinoamericanos y también norteameri-canos y europeos (Menéndez-Carrión, 1991a).

FLACSO mantiene seis proyectos de investigación sobre Amazonia, centrados en estudios comparativos de África, Asia y América Latina (Amazonia), modelos cognoscitivos de manejo de la naturaleza durante los procesos de colonización, ecodesarrollo participativo, definición y manejo de zonas de reserva ecológica, creación de una base de datos sobre la Amazonia y cronología de acontecimientos relativos a la región.

FLACSO cuenta con 10 profesores/investigadores de planta, seis profesores/investigadores asociados y un gran numero de profesores visitantes - en el año 1990-1991 llegó a 46 -, además de asistentes de docencia, investigadores asistentes, ayudantes de investigación e investigadores invitados. Entre 1988 y 1991 se ejecutaron 12 programas académicos, atendiendo un total de 254 alumnos; se congregaron 95 profesores visitantes; se ofrecieron 135 eventos nacionales e internacionales; atendieron a las actividades de la facultad mas de 300 académicos provenientes de América Latina, Norteamérica y Europa; 
se recibieron y procesaron más de 50 peticiones de cooperación técnica, de las cuales 12 se constituyeron en programas de investigación o de discusión específicos; y se firmaron 34 convenios de cooperación con organismos nacionales e internacionales. Entre 1987 Y 1991 se publican 16 libros, 20 documentos de trabajo y 12 trabajos diversos (MENENDENDEZ- CARRIÓN, 1991b).

Los estudios amazónicos son recientes en FLACSO y por lo tanto su desarrollo es aún tenue en comparación a áreas más consolidadas. Sin embargo, su alcance y proyección son promisores en el universo de actividades que desarrolla la institucion. La concentración de intereses en la realidad andina permite al investigador de Amazonia analizarla de manera integrada, es decir, considerando las influencias y/o reproducción de los procesos sociales de los Andes en la Amazonia y el impacto que el desarrollo de esta región puede tener en las modificaciones socioeconómicas de los Andes. De la misma forma, por su carácter internacional, el curso de maestría con énfasis en estudios amazónicos permitirá entender la Amazonia a nivel continental y desarrollar estudios comparativos entre las diferentes regiones de los países amazónicos.

Las ciencias sociales aplicadas a Amazonia tanto en investigación como en programas de formación de recursos humanos serán ampliadas en Ecuador y en los países andinos (principalmente) a partir del retorno de los magisters egresados de FLACSO, ya que su clientela mayor proviene de universidades, institutos de investigación y organismos de planificación. Por otro lado, actividades como las que desarrolla FLACSO permiten al Ecuador afirmarse cada vez mas como país amazónico.

\section{BOLIVIA}

Tradicionalmente Bolivia ha orientado su investigación a temas relacionados con el altiplano y valles, principalmente sobre minería y agricultura de subsistencia. A partir de 1950, pero especialmente durante la década de los setenta, la Amazonia toma relevancia en las investigaciones científicas relacionadas con la ampliación de la frontera, el avance de la economía de la coca, la búsqueda de nuevos productos para el mercado nacional y la diversificación de exportaciones con productos provenientes del trópico húmedo (BLANES, 1988).

La gran mayoría de temas de la bibliografía amazónica se concentra en ciencias sociales, cubriendo tópicos sobre colonización, pecuaria y explotación de recursos naturales, y las investigaciones son realizadas principalmente por el Centro Internacional de Agricultura Tropical, por 
instituciones gubernamentales y por instituciones internacionales. La universidad aparentemente no tiene mayor influencia en la investigación amazónica, por lo menos hasta 1986.

Por otro lado, es interesante ver que la producción bibliográfica no coincide con las temáticas de los proyectos de investigación registrados en la Dirección Científica y Tecnológica del país. De los 193 proyectos científicos registrados por esa entidad en 1986, 14 se refieren a la Amazonia, centrados en temas de desarrollo experimental, utilización de recursos naturales, fauna, climatología y balance hídrico; solamente uno toca la problemática social (mejoramiento de la tecnología nativa, ejecutado en Santa Cruz por el Proyecto de Desarrollo Agrícola Integrado, en convenio con el Instituto Interamericano de Cooperación para la Agricultura y la Corporación de Desarrollo de Potosí) (BLANES, 1988).

Según Blanes (1988), 55 instituciones realizan investigación científica en Bolivia, concentradas en los departamentos de La Paz, Cochabamba y Santa Cruz, siendo que $48 \%$ de ellas son universidades. De las ocho universidades nacionales existentes en el país, dos se Iocalizan en la Amazonia: la Universidad Autónoma Gabriel René Moreno, en Santa Cruz de la Sierra, y la Universidad Técnica del Beni Mariscal J ose Ballivian, en Trinidad. Una novena universidad, la Universidad de Pando, en Cobija, plena Amazonia, fue creada a inicios de la década de los ochenta pero hasta ahora no ha sido instalada.

La formación de recursos humanos en las universidades bolivianas sigue la escuela tradicional, con la mayoría de graduados en las carreras de medicina, derecho y más recientemente agronomía. Las propias universidades amazónicas no surgieron de planes deliberados de desarrollo de la región y, por consiguiente, ellas no se diferencian de las del resto del país. En diez años (1972-1982), por ejemplo, las universidades amazónicas escasamente alcanzaron a acumular diez mil matrículas en agronomía, en relación a números mucho más elevados en medicina, y jurisprudencia. Es también importante destacar que la agronomía y otras carreras que se suministran en esas universidades, como lo demuestran los proyectos que ellas vienen desarrollando, no se relacionan necesariamente a la región amazónica (BLANES, 1988).

El impulso dado por la Asociación de Universidades Amazónicas (UNAMAZ) en la Universidad Autónoma Gabriel René Moreno ha sido importante para el avance de las actividades relacionadas con la investigación amazónica en Bolivia. Además de los cursos de postgrado en desarrollo agrario y economía internacional que ofrece desde 1989, la universidad ha aumentado considerablemente su participación en 
seminarios, conferencias, cursos de especialización, inclusive en la organización de muchas de estas actividades, desde la fundación de la UNAMAZ en 1987.

\section{VENEZUELA}

El desarrollo científico y tecnológico en Venezuela ha sido determinado por la economía derivada de la explotación petrolera. En general, los recursos generados por esa actividad atrasaron la formulación de políticas científicas y tecnológicas a ser desarrolladas nacionalmente, ya que tal economía permitía importar tecnología o formar recursos humanos en el exterior. Sólo a partir de 1967, cuando se crea el Consejo Nacional de Investigaciones Científicas y Tecnológicas (CONICIT) es que se comienzan a estructurar planes de desarrollo en el área científica y tecnológica para el país. Pero inclusive con la creación del CONICIT, las políticas estaban orientadas principalmente a desarrollar y fortalecer tecnologías vinculadas a la explotación petrolera. Venezuela invierte $1,57 \%$ del presupuesto nacional (1990) en ciencia y tecnología, cuenta con 5.500 científicos y tecnólogos, 800 unidades de investigación y más de 7.000 proyectos de investigación en curso (SANTANA NAZOA, 1991, p. 108).

Más del $60 \%$ de los investigadores han realizado estudios de postgrado y están concentrados en las universidades, principalmente en aquellas localizadas en las regiones central y de los Andes. Estas dos regiones absorben $80 \%$ de los investigadores, lo que deja en condiciones extremamente desfavorables a la región amazónica venezolana. Pese a la existencia de esta masa crítica, el CONICIT reconoce que la deficiencia de investigadores en el país llegaba en 1990 a 13.788, o sea de 72\% (SANTANA NAZOA, 1991, p. 108).

No existe a nivel nacional un plan de desarrollo científico y tecnológico para la Amazonia venezolana, lo que en la visión de algunos científicos nacionales representa seria amenaza para el desarrollo y el ejercicio de la propia soberanía nacional, que podrá generar efectos irreversibles en el ambiente y las sociedades que habitan la región (SANTANA NAZOA, 1991; BARRETO LOPEZ, 1992).

A nivel local, existe un Plan Estatal de Desarrollo del Territorio Federal Amazonas (1991-1993), donde se contempla el área de ciencia y tecnología, enfatizando principalmente el fortalecimiento de la infraestructura en el territorio, necesaria para iniciar proyectos de investigación científica y programas que promuevan el desarrollo regional. 
Llevando en cuenta esas consideraciones, no es sorprendente que la investigación científica en general y la social en particular sobre la Amazonia venezolana esté asociada al esfuerzo de unas pocas instituciones y algunos investigadores con interés particular en la región. En base a dos trabajos bibliográficos, Santana Nazoa (1991) consigue realizar una primera aproximación de la producción científica de acuerdo a diferentes tópicos y ramas del saber. La obra de Weibezahn $\&$ J anssen (1990) registra 1.469 entradas bibliográficas. La mayoría de títulos se refiere a medio ambiente y manejo de recursos naturales, antropología de grupos aborígenes, salud y medicina tropical, y descripción física y cultural de la región. Son escasas las referencias sobre recursos minerales y estratégicos, demografía y aspectos poblacionales, etnobotánica, plantas medicinales, educación, fronteras, seguridad y defensa, geopolítica, inventario de recursos, hidroeléctricas, planificación y producción, y predominan las publicaciones en el exterior.

El segundo trabajo bibliográfico elaborado por Moreno \& Gonzalez (1989) incluye 498 referencias, siendo que su distribución por áreas temáticas coincide bastante con la obra de Weibezahn \& J anssen (1990). Geografía e historia representan $49 \%$ de las referencias, donde se incluyen temas de geografía física y se nota la baja proporción (2\%) de referencias de educación y lingüística. De igual forma prevalece un alto porcentaje (14\%) de publicaciones realizadas por universidades extranjeras (SANTANA NAZOA, 1991, p. 166-167).

Según el levantamiento realizado por Santana Nazoa (1991) sobre las instituciones, investigaciones e investigadores que desarrollan proyectos acerca de la Amazonia, las proporciones que ellos representan a nivel nacional son extremamente pequeñas: 15 instituciones, o 2\% del total nacional; 164 investigaciones, o 2\% del total nacional; y 71 investigadores, $01 \%$ del total nacional. Solamente 5 de las 15 instituciones que realizan investigación amazónica tienen su sede en el Amazonas. Como consecuencia, las investigaciones son lideradas por aquellas instituciones que están localizadas fuera de la Amazonia venezolana, principalmente por el Instituto Venezolano de Investigaciones Científicas (IVIC), y por la Universidad Central de Venezuela (UCV) (a través del Proyecto A mazonas principalmente). En la Amazonia se realizan $27 \%$ de las investigaciones, lideradas por el Centro Amazónico para Investigaciones y Control de Enfermedades Tropicales Simón Bolívar (CAICET), en el área de salud. En efecto, esta es la única área en que se hace más investigación sobre A mazonia dentro que fuera de ella. Como es de esperarse, la mayoría de investigadores 
(93\%) están radicados en lugares fuera de la Amazonia, especialmente en instituciones de Caracas. Un factor importante a considerar es que solamente $59 \%$ de los investigadores tienen curso de postgrado (de especialización a doctorado), distribuidos $7 \%$ residentes en el Amazonas y $52 \%$ en el resto del país. Cuando se consideran estrictamente los 18 investigadores que viven en el A mazonas venezolano, solamente 5 poseen algún tipo de curso de postgrado (2 especialización, 2 maestría y 1 doctorado).

Considerando las áreas temáticas del Plan de Acción en Ciencia y Tecnología del Tratado de Cooperación Amazónica, se nota claramente la predominancia de la investigación en el área del medio ambiente y manejo de recursos naturales, confirmando los levantamientos bibliográficos analizados arriba. La investigación social podría ubicarse principalmente en el área de poblaciones humanas, la segunda en importancia de acuerdo al levantamiento de Santana Nazoa (1991). Esto representa principalmente la acción del IVIC, de la UCV y de la Fundación La Salle de Ciencias Naturales; y dentro de la Amazonia, la del Servicio Autónomo para el Desarrollo Ambiental del Amazonas, del Museo Etinológico de Puerto Ayacucho Enzo Ceacarelli, y del Centro al Servicio de la Acción Popular. Las investigaciones sobre poblaciones humanas se concentran principalmente en etnología y etnolingüística (14 investigaciones) y temas relacionados a la problemática indígena, descuidando otros cruciales, como la colonización y la urbanización, que no presentan ningún registro, o los impactos socioculturales de los planes de desarrollo que registran solamente 3 proyectos.

En lo que se refiere a la capacitación de recursos humanos, ésta queda por cuenta de algunas carreras que incluyen en sus currículos disciplinas que de alguna manera se relacionen con la Amazonia. No existen cursos permanentes a nivel de postgrado que capaciten a especialistas en asuntos amazónicos, aunque el país posee infraestructura para tal. Tal vez en el área de salud ${ }^{6}$ se toquen los problemas de enfermedades tropicales en algunas universidades y en el propio CAICET, pero en ciencias sociales no se conoce especialidad en estudios amazónicos. Esto quiere decir que la mayoría de especialistas en estudios amazónicos que laboran en Venezuela han sido formados en el exterior, o de alguna manera se han interesado personalmente por esos asuntos.

\footnotetext{
${ }^{6}$ Un ejemplo importante en el área de salud es el proyecto sobre Evaluación de Impactos sobre Salud Ambiental en la Amazonia, que desarrolla la UNAMAZ, con la participación de la Universidade Federal de Mato Grosso, en Brasil, la Universidad de los Andes, en Colombia, y la Universidad Central de Venezuela, en Venezuela, bajo la coordinación de esta última.
} 


\section{GUYANAS}

Con la participación de Guyana y Surinam del Tratado de Cooperación Amazónica en 1978, el carácter amazónico de esos dos países aflora, aumentando sus intercambios y cooperación técnica con los demás países del Tratado (SAUL, 1989).

Como consecuencia de su participación en varias actividades del Tratado, Guyana y Surinam están aumentando sus programas de formación de recursos humanos e investigación amazónica. La Universidad de Guyana, creada en 1963, mantiene actualmente siete facultades, cerca de 2.300 estudiantes y 500 profesores. La universidad realiza investigación amazónica desde hace cerca de 10 años, sobre flora, fauna y, más recientemente, sobre manejo y planificación ambiental, ecología, educación a distancia y problemas de los grupos aborígenes este último a través del programa de estudios amerindios en la Facultad de Artes (WALCOTT, 1988).

Por su parte, la Universidad de Surinam, fundada en 1968, tenía en 1990, 1.900 estudiantes y 223 profesores/investigadores, distribuidos en tres facultades. No existe un programa específico de estudios amazónicos. La investigación relacionada con la región se realiza a criterio de algunos investigadores interesados en las propias facultades, especialmente sobre medicina, biología y minerales no metálicos (BRUNINGS, 1988).

\section{CONCLUSIÓN}

Es extremamente difícil trazar un perfil de la situación de la investigación en ciencias sociales aplicadas a Amazonia en los países que integran la cuenca, en base a los datos disponibles para este estudio. Las informaciones contenidas en las referencias cubren universos y periodos diferentes. Por ejemplo el trabajo de Aragón (1990) se refiere solamente a la investigación realizada en la Amazonia brasileña, dejando de lado instituciones importantes que realizan investigación amazónica pero que se localizan fuera de la región, mientras que los estudios de Mora \& Zarzar (1990) y Correa (1990) cubren la investigación amazónica hecha en todo el país, Peru y Colombia respectivamente. Por su parte, Mora \& Zarzar (1990) se refieren estrictamente a la investigación en ciencias sociales y humanas, cubriendo el período 1988-1989. Otros dos autores, Correa (1990) y Aragón (1990), cubren períodos mayores y cubren también, con fines comparativos, investigación amazónica en otras ramas del saber. Las informaciones referentes a los demás países amazónicos son aún más incompletas. 
Pese a esas limitaciones se pueden hacer algunas afirmaciones a nivel de la Amazonia continental. Por lo menos 150 instituciones realizan investigación o producen ciencia y tecnología relacionada con Amazonia en los ocho países, de las cuales aproximadamente 100 hacen investigación de tipo social. Esas instituciones están concentradas mayormente en Colombia y Brasil. Por lo menos 500 investigaciones en el área de ciencias sociales y humanas referentes a la Amazonia están en curso y el número de investigadores de ciencias sociales realizando investigación amazónica puede llegar a 800, cerca de la mitad actuando dentro de la Amazonia brasileña. La mayoría de instituciones (excepto en Brasil) que hacen investigación amazónica se localiza fuera de la región, concentrándose en las capitales nacionales.

Los países presentan desarrollo desigual en lo que atañe a la producción científica y tecnológica referente a la Amazonia. En este sentido, la antropología fue la disciplina pionera en los estudios amazónicos en todos los países. La Amazonia ha contribuido de esta manera al avance teórico de las ciencias sociales, pero esa producción científica poco ha sido utilizada para el desarrollo integral y equilibrado de la región. A nivel estatal todavía se considera el problema del desarrollo amazónico como un problema eminentemente técnico, donde supuestamente las ciencias sociales tendrían poco a contribuir.

En lo que se refiere a programas de formación de recursos humanos, en la mayoría de países los estudios amazónicos son incorporados en asignaturas de algunas carreras, como antropología, sociología e historia que incluyen temas amazónicos como parte del currículo. A nivel de postgrado, solamente Brasil y Ecuador mantienen cursos de maestría con concentración en estudios amazónicos, los dos de carácter interdisciplinar, el primero concentrado en planificación del desarrollo, de ámbito panamazónico, y el segundo concentrado en ciencias sociales, de ámbito esencialmente andino. Sin embargo, la mayoría de países cuenta hoy con infraestructura institucional básica en términos de cantidad de establecimientos para aumentar la masa crítica que está en formación.

Es evidente que las acciones que se realizan en investigación y formación de recursos humanos referentes a Amazonia obedecen a intereses nacionales, sin mayor integración o coordinación regional. Es necesario crear o fortalecer mecanismos que integren estos esfuerzos aislados en programas cooperativos para maximizar los escasos recursos financieros y humanos disponibles, tal como se comienza a trabajar en Brasil a través de la CORPAM y en Colombia a través del CONIA. Redes de investigadores, a ejemplo de la Red de Investigadores de la Amazonia 
Peruana, y redes de instituciones, a ejemplo de la UNAMAZ, son instrumentos esenciales para fomentar el trabajo interdisciplinar. Esfuerzos como los que realiza el NAEA en Brasil y la FLACSO en Ecuador deben analizarse con cuidado y considerarse seriamente en cualquier programa de cooperación internacional en el área de ciencias sociales. En este sentido, la Comisión de Ciencia y Tecnología del Tratado de Cooperación Amazónica juega papel importante, especialmente a través de sus programas de Política de Ciencia y Tecnología para la Amazonia y de Estudios e Investigaciones en Ciencias Sociales. La ejecución de esos programas debe partir de programas de investigación y formación de recursos humanos que vienen siendo ejecutados aisladamente por los países. 


\section{REFERENCIAS}

ALZATE, Beatriz; RAMíREZ, María Tereza V. 1991. Cinco lustros de actuación institucional nacional e internacional en Amazonia. Bogotá: Ministerio de Educación Nacional.

ARAGÓN, Luis E. 1990. Investigación de ciencias sociales en la Amazonia brasileña. Belém (mimeo.).

. 1994. The Amazon as a study object: building regional capacity for sustainable development. Stockholm: Institute of Latin American Studies/Stockholm University.

. 1995. Building regional capacity for sustainable development in the Amazon. In: D'AVILA NETO, Maria Inácia (org.). Social development: challenges and strategies. Rio de J aneiro: UNESCO Chair on Sustainable Development. p. 275-325.

AZEVEDO, Aline da Rin Paranhos de. 1989. Informação e documentação científica, tecnológica e cultural na Amazônia brasileira. In: ARAGÓN, Luis E., IMBIRIBA, Maria Nazaré (org.). Informação e documentação na Amazônia. Belém: UFPA. p. 13-28.

BARRETO LÓPEZ, Sergio Rafael. 1992. La planificación del Estado y las políticas de desarrollo regional y científico-tecnológicas para el Amazonas venezolano. In: BELTRÃO, J imena Felipe, VILLAS, Raimundo Netuno Nobre (org.). Ciência e tecnologia: desafio amazônico. Belém: UNAMAZ/UFPA. p. 85-120.

BARROS, Fernando, BENEDITO, J ose Edil. 1990. A questão institucional da ciência e tecnologia na Amazônia. In: BARROS, Fernando A. F. (coord.). C \& $T$ no processo de desenvolvimento da região amazônica. Brasília: CNPq/PTU. v. I, p.11-67.

BEDOYA, Roberto. 1989. La formación de profesores indígenas mediante el sistema de estudio a distancia y bilingüe bicultural de la UNAP. In: ARAGON, Luis E., IMBIRIBA, Maria Nazaré (org.). Informação e documentação na Amazônia. Belém: UFPA. p. 161-64.

BENEDITO, José Edil (coord.). 1990. Subsídios à CORPAM para a montagem da programação de pesquisa na Amazônia. Brasília: CNPq (mimeo.).

BERQUÓ, Elza. 1983. Demografia. In: CNPq. Avaliação e perspectivas. Brasília: CNPq. 
. 1988. ABEP - Primeira década: avanços, lacunas e perspectivas. Belo Horizonte: Associação Brasileira de Estudos Populacionais (ABEP).

BLANES, J osé. 1988. Bolivia, el papel de la universidad en el desarrollo científico y tecnológico de la región amazónica. In: ARAGÓN, Luis E., IMBIRIBA, Maria Nazaré (org.). Universidade e desenvolvimento amazônico. Belém: UFPA. p. 61-122.

BRASIL. Ministério da Educação. 1992. Sinopse estatística do ensino superior-graduação. Brasília.

BRUNINGS, Ernie. 1988. The impact of university and research institutes on scientific and technological development: the case of Suriname, with special references to the amazonian region. In: ARAGÓN, Luis E., IMBIRIBA, Maria Nazaré (org.). Universidade e desenvolvimento amazônico. Belém: UFPA. p. 355-414.

CHIRIF, Alberto. 1991. Investigación para el desarrollo. In: RUIZ, Lucy (org.). Amazonia nuestra: una visión alternativa. Quito: CEDIME. p. 269-85.

CORPAM. 1990. Plano de Ciência e Tecnologia para a Amazônia, 90/ 91. Belem: UFPA.

CORREA, Francisco. 1990. Investigación de ciencias sociales en la Amazonia colombiana. Bogotá (mimeo.).

DEAL, Carl W. 1977. Latin America and the Caribbean: a dissertation bibliography. Ann Arbor: University Microfilms International.

DURHAM, Eunice Ribeiro. 1991. Formación de recursos humanos de alto nivel: Ias nuevas funciones de los postgrados. In: CRESALC. Retos científicos y tecnológicos. Caracas: CRESALC/UNESCO. p. 99-123.

FIGUEIREDO, Napoleão, BRUNI, Sérgio de Almeida. 1987. Museu Paraense Emílio Goeldi: 120 anos na busca da excelência científica. Belém: MPEG.

FLACSO. 1991a. Memoria 1989-1990. Quito.

. 1991b. Informe de actividades, enero a octubre 1991. Quito.

INFORMAM. 1987. Cadastro de pesquisas sobre a Amazônia brasileira. Belém: UFPA. 4 v.

1988. Cadastro de instituições de pesquisa da Amazônia brasileira. Belém: UFPA. 
MENDES, Armando Dias. 1983. NAEA: nos primórdios. Brasília (mimeo.).

MENÉNDEZ-CARRIÓN, Amparo. 1991a. 1989-1990: dos años de logros, expansión y nuevos desafíos. In: FLACSO. Memoria 1989-1990. Quito. p. 19-32.

$\overline{1} \overline{9} \overline{9} \overline{1}$. Quito.

1991b. Informe de gestión, septiembre 1987 a septiembre

MORA, Carlos. 1988. Ciencia, tecnología y sociedad en la Amazonia del Perú. In: ARAGÓN, Luis E., IMBIRIBA, Maria Nazaré (org.). Universidade e desenvolvimento amazônico. Belém: UFPA. p.317-53.

MORA, Carlos, ZARZAR, Alonso. 1990. Las ciencias sociales y la Amazonia en el Peru. Lima (mimeo.).

MORENO, Celsa, GONZÁLEZ, Marisol. 1989. Aportes para la recuperación de la bibliografia del Amazonas. Tesis de grado, Escuela de Bibliotecología, Universidad Central de Venezuela,Caracas.

MPEG (Museu Paraense Emílio Goeldi). 1989. Relatório de atividades: 1988. Belém.

OLIVEIRA, Adélia Engrácia de. 1983. Ocupación humana. In: SALATI, Eneas et al. (org.). Amazônia: desenvolvimento, integração e ecologia. São Paulo: CNPq/Brasiliense. p. 144-327.

SANTANA NAZOA, Aída. 1991. La cuestión científica y tecnológica en el Amazonas venezolano: evaluación y perspectivas. Tesis de maestría, Centro de Estudios del Desarrollo, Universidad Central de Venezuela, Caracas.

SANTOS GRANERO, Fernando. 1991. La historiografía amazónica en perspectiva: 1950-1990. In: RUIZ, Lucy (org.). Amazonia nuestra: una visión alternativa. Quito: CEDIME. p. 49-93.

SAUL, Compton. 1989. Population and amazonian development: the case of Guyana.In: ARAGÓN, Luis E., IMBIRIBA, Maria Nazaré (org.). Populações humanas e desenvolvimento amazônico. Belém: UFPA. p. 193-265.

SAWYER, Donald. 1989. Inventário e avaliação da produção técnicocientífica sobre migração na Amazônia Legal. Belém: SUDAM/UFMG/ CEDEPLAR/FUNDEP.

UFPA (Universidade Federal do Pará). 1988. Memória do I Seminário de Avaliação da Pesquisa na UFPA. Belém. 
VALENCIA GRANADA, Alberto. 1988. Papel de la universidad y de los institutos de investigación en el desarrollo científico-técnico de la región amazónica colombiana. In: ARAGÓN, Luis E., IMBIRIBA, Maria Nazaré (org.). Universidade e desenvolvimento amazônico. Belém: UFPA. p. 123-95.

WALCOTT, George. 1988. University of Guyana perspectives in relation to a programme of international and interinstitutional cooperation to promote the scientific and technological development of the Amazon region. In: ARAGÓN, Luis E., IMBIRIBA, Maria Nazaré (org.). Universidade e desenvolvimento amazônico. Belém: UFPA. p. 291-316.

WEIBEZAHN, Frany, JANSSEN, W. Bárbara. 1990. El Territorio Federal Amazonas. Venezuela: uma bibliografía. Caracas: CONICIT.

YANEZ, Segundo Moreno. 1991. Corrientes antropológicas en los estudios sobre la Amazonia ecuatoriana. In: RUIZ, Lucy (org.). Amazonia nuestra: una visión alternativa. Quito: CEDIME. p.95-103.

ZUQUILANDA DUQUE, Patricio. 1988. Papel de las universidades e institutos de investigación en el desarrollo científico-técnico de la región amazónica ecuatoriana. In: ARAGÓN, Luis E., IMBIRIBA, Maria Nazaré (org.). Universidade e desenvolvimento amazônico. Belem: UFPA. p. 197290. 
Luis E. Aragon

Texto encomendado pela Revista em 18/07/2004 e aceito para publicação em 30/07/2004 66 